\title{
Função discriminante de Fisher para classificação do carvão vegetal de clones de Corymbia e Eucalyptus
}

\author{
Fisher's discriminant function for classification of the charcoal of Corymbia and \\ Eucalyptus clones
}

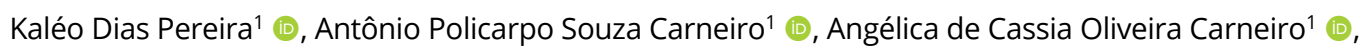
Gerson Rodrigues dos Santos ${ }^{1}$ (D), Lucas de Freitas Fialho ${ }^{1}$ (1)

${ }^{1}$ Universidade Federal de Viçosa - UFV, Viçosa, MG, Brasil

Como citar: Pereira, K. D., Carneiro, A. P. S., Carneiro, A. C. O., Santos, G. R., \& Fialho, L. F. (2021). Função discriminante de Fisher para classificação do carvão vegetal de clones de Corymbia e Eucalyptus. Scientia Forestalis, 49(130), e3474. https://doi.org/10.18671/scifor.v49n130.07

\begin{abstract}
Resumo
Objetivou-se utilizar a função discriminante de Fisher para sumarizar as propriedades do carvão vegetal e, assim, classificar clones do gênero Corymbia e Eucalyptus quanto ao rendimento e qualidade da produção de carvão. Foram consideradas seis variáveis: rendimento da carbonização, teor de materiais voláteis, carbono fixo, friabilidade, densidade relativa aparente e poder calorífico superior do carvão vegetal de onze clones de Corymbia e um de Eucalyptus. Os dados foram previamente testados quanto às pressuposições de normalidade multivariada e homogeneidade de variâncias/covariâncias. Posteriormente, foi realizada a análise de variância multivariada e aplicada a função discriminante de Fisher para transformação das variáveis originais em duas variáveis canônicas. A comparação dos clones quanto ao rendimento e qualidade do carvão vegetal foi realizada aplicando-se o teste de Scott-Knott a $1 \%$ de significância sobre as médias das variáveis canônicas. Em comparação às análises univariadas realizadas, o uso da função discriminante de Fisher para gerar novas variáveis apresenta vantagens quanto a simplificação da interpretação dos resultados e, principalmente, na discriminação dos clones em relação à qualidade global do carvão vegetal. Comparando os clones por meio das variáveis canônicas constatou-se que o clone Eucalyptus grandis x Eucalyptus urophylla é o mais indicado para a produção de carvão vegetal. Não obstante, os clones de Corymbia apresentaram desempenho promissor para utilização na produção de carvão.
\end{abstract}

Palavras-chave: Análise multivariada; Corymbia; Eucalyptus; Redução de dimensionalidade; Variável canônica.

\begin{abstract}
The objective of this study was to use Fisher's discriminant function to summarize charcoal properties and classify clones of Corymbia and Eucalyptus genus regarding yield and quality of charcoal production. Six variables were considered: carbonization yield, volatile matter content, fixed carbon, friability, apparent relative density and higher calorific value of charcoal from eleven clones of Corymbia and one clone of Eucalyptus. The data were previously tested regarding multivariate normality and variances/covariances homogeneity. Subsequently, the multivariate analysis of variance was performed and Fisher's discriminant function was applied to transform the original variables into two canonical variables. The comparison of the clones towards the charcoal yield and quality was realized by applying the Scott-Knott's test at $1 \%$ significance with the averages of the canonical variables. Different than the univariate analysis realized, the use of the Fisher's discriminant function to generate new variables presents advantages in terms of interpretation simplifying of the results and mainly in the differentiation of the clones in relation to the general charcoal quality. Comparing the clones by canonical variables
\end{abstract}

Fonte de financiamento: Coordenação de Aperfeiçoamento de Pessoal de Nível Superior - CAPES, código de financiamento 001.

Conflito de interesse: Nada a declarar.

Autor correspondente: kaleoef@gmail.com

Recebido: 16 setembro 2019.

Aceito: 22 abril 2020.

Editor: Paulo Henrique Müller Silva.

(c) (i) Este é um artigo publicado em acesso aberto (Open Access) sob a licença Creative Commons Attribution, que permite uso, distribuição e

ceprodução em qualquer meio, sem restrições desde que o trabalho original seja corretamente citado. 
averages, it was found that the Eucalyptus grandis $x$ Eucalyptus urophylla clone is the most suitable for charcoal production. Notwithstanding, the clones of the genus Corymbia had a promising performance for utilization in charcoal production.

Keywords: Multivariate analysis; Corymbia; Eucalyptus; Dimensionality reduction; Canonical variable.

\section{INTRODUÇÃO}

De acordo com o último relatório elaborado pela FAO (Food and Agriculture Organization, 2017), o Brasil é o maior produtor de carvão vegetal do mundo, produzindo 6,2 milhões de toneladas em 2015, o que representou cerca de $12 \%$ da produção global daquele ano. O carvão vegetal consumido dentro do país é destinado principalmente às indústrias de ferrogusa, ferro-liga e aço, chegando a um consumo interno de 4,5 milhões de toneladas de carvão em 2017, fazendo do Brasil líder mundial na produção de aço a partir de carvão vegetal, que se caracteriza por ser uma liga metálica de melhor qualidade e com menor impacto ambiental durante a produção (Associação Brasileira de Produtores de Florestas Plantadas, 2013; Indústria Brasileira de Árvores, 2018).

As florestas de eucalipto têm sido as mais utilizadas para a produção de carvão, visto que apresentam alta produtividade volumétrica e madeira com características apropriadas para este fim (Goulart et al., 2003). Devido à variabilidade da qualidade do carvão vegetal e à exigência do mercado, é importante que as empresas de reflorestamento busquem selecionar materiais genéticos mais produtivos e com propriedades da madeira favoráveis à qualidade do carvão (Trugilho et al., 2001; Oliveira et al., 2010; Castro et al., 2013).

Os estudos com qualidade do carvão vegetal se deparam com um amplo conjunto de variáveis, o que direciona frequentemente tais pesquisas às análises com técnicas multivariadas (Protásio et al., 2012, 2013; Castro et al., 2013). A vantagem da utilização de técnicas multivariadas, segundo Mingoti (2005), deve-se às dependências que normalmente existem entre as variáveis, fato que torna as análises univariadas dispendiosas e, ocasionalmente, com resultados controversos.

Quando uma pesquisa é planejada para selecionar clones, o objeto foco do experimento é definido como sendo um fator qualitativo em que seus níveis são diferentes genótipos, ou seja, o objetivo é comparar diferentes clones em função de uma ou mais variáveis. As análises multivariadas apresentam algumas alternativas que podem simplificar a comparação de médias partindo de um grande conjunto de variáveis observadas, como análise de componentes principais, análise de fatores e análise de variância multivariada (MANOVA). Gomes (2009) descreveu uma proposta de aplicação da função discriminante de Fisher ou primeira variável canônica que, diferente do uso convencional da análise discriminante para classificação de um indivíduo em uma população predefinida (Fisher, 1936), possibilita transformar todas as variáveis originais em uma única variável que retém a maior parte da informação dos dados.

A utilização da função discriminante como complemento da MANOVA para transformar as variáveis sem grande perda de informação resulta numa simplificação eficiente para interpretação dos resultados (Campos, 2012). No contexto da avaliação das propriedades do carvão, a função discriminante conforme a metodologia descrita por Gomes (2009) é um meio de resumir toda a informação em um único indicador de qualidade global do carvão vegetal que é uma função das variáveis observadas. Sendo assim, objetivou-se utilizar a função discriminante de Fisher para sumarizar as propriedades do carvão vegetal e, assim, classificar clones do gênero Corymbia e Eucalyptus quanto ao rendimento e qualidade da produção de carvão.

\section{MATERIAL E MÉTODOS}

Os dados foram coletados em plantios localizados no Município de Dionísio, Minas Gerais. O clima da região é classificado como subtropical úmido, cuja temperatura média anual varia entre 20 e $23^{\circ} \mathrm{C}$ e a precipitação média é de 1100 a $1400 \mathrm{~mm}$ (Motta et al., 1996). O plantio de onze clones de Corymbia torelliana x Corymbia citriodora e um de Eucalyptus 
grandis x urophylla, foi realizado em 2008, em área de Latossolo Vermelho-Amarelo com espaçamento entre plantas de $3 \times 2,5 \mathrm{~m}$.

Os plantios tinham 7 anos de idade quando as amostras para as análises das propriedades do carvão foram coletadas. Foi determinado o rendimento gravimétrico, densidade relativa aparente, friabilidade, teor de materiais voláteis, teor de carbono fixo e poder calorífico superior do carvão vegetal conforme as metodologias descritas por Vital (1984), Oliveira et al. (1982), NBR 8112 (Associação Brasileira de Normas Técnicas, 1986) e NBR 8633 (Associação Brasileira de Normas Técnicas, 1984).

A avaliação das diferenças entre os atributos de qualidade do carvão entre os diferentes clones foi realizada por meio da MANOVA. Precedendo a realização da MANOVA, os dados foram submetidos a análise de normalidade e homocedasticidade de matrizes de variâncias/covariâncias.

A normalidade dos dados foi avaliada inicialmente pelo teste de Shapiro-Wilk a $1 \%$ de significância aplicado à cada variável individualmente. A normalidade multivariada foi avaliada por meio dos testes de assimetria e curtose propostos por Mardia (1970,1974, 1975) a 1\% de significância. Para a avaliação univariada das variáveis quanto a homocedasticidade de variâncias foi aplicado o teste de Bartlett a $1 \%$ de significância. A generalização do teste de Levene com o método PERMDIST (Anderson, 2006), foi utilizado para analisar a homogeneidade de matrizes de variância/covariância. Também foi realizada uma análise para detecção de outliers multivariados pelo método da distância de Mahalanobis robusta.

A análise de variância multivariada (MANOVA) foi realizada conforme Gomes (2009), utilizando o critério lambda de Wiks $(\Lambda)$ com aproximação para a distribuição $F$ para testar a hipótese de igualdade dos vetores de médias dos clones $\left(\mathrm{H}_{0}: \mu_{\mathrm{v} 1}=\mu_{\mathrm{v} 2}=\ldots=\mu_{\mathrm{v} 12}\right)$, conforme a transformação indicada para casos gerais de graus de liberdade (Harris, 1975). Em adição, também foram realizados os testes de Pillai, de Hotelling-Lawley e de Roy para efeito de ratificação.

A aplicação da função discriminante para gerar uma variável canônica em função das variáveis originais foi realizada conforme descrito por Gomes (2009), o que indica a utilização da primeira função discriminante baseado na ideia de reter a maior parte da informação contida nos dados e maximizar a discriminação dos tratamentos, visto que esta é associada ao maior autovalor. A retenção de informação foi mensurada pela proporção do autovalor em relação a soma de todos os autovalores.

Os coeficientes da função discriminante foram estimados a partir da decomposição espectral da matriz $\mathbf{E}^{-1} \mathbf{H}$, sendo $\mathbf{E}$ a matriz de somas de quadrados e produtos cruzados do erro e $\mathbf{H}$ a matriz de soma de quadrados e produtos cruzados dos clones. A normalização de cada coeficiente foi realizada conforme a equação:

$$
b_{p}^{*}=\frac{b_{p}}{\sqrt{\sum_{p=1}^{6}\left(b_{p}\right)^{2}}},
$$

em que $b_{p}^{*}$ é o coeficiente normalizado e $b_{p}$ o não normalizado. Estimada a variável canônica, submeteu-se a nova variável ao teste de normalidade de Shapiro-Wilk e ao teste de homocedascidade de Bartlett, ambos a 1\% de significância. Em seguida, a variável canônica foi submetida à ANOVA e os clones foram comparados por meio da variável canônica aplicando-se o teste de Scott-Knott a 1\% de significância.

Para validar e comparar a qualidade da função discriminante em relação às análises univariadas tradicionais, foram utilizados critérios similares ao de Campos (2012). O principal critério de comparação foi a magnitude da estatística $F$ e os respectivos valores-p, além do cálculo da acurácia seletiva (Resende, 2002): 
$\mathrm{AS}=\left(1-\frac{1}{\mathrm{~F}}\right)^{1 / 2}$

em que AS é a acurácia seletiva, F é a razão entre quadrado médio dos clones e quadrado médio do resíduo. Para a realização das análises foram utilizados os pacotes vegan, MVN, car e ScottKnott do R (R Core Team, 2018).

\section{RESULTADOS E DISCUSSÃO}

Apesar da análise de normalidade multivariada ter indicado rejeição da hipótese nula de simetria, para a curtose houve aproximação da normal multivariada. Analisando a normalidade das variáveis individualmente, constatou-se distribuição normal em quase todos os casos, apenas o teor de materiais voláteis não apresentou distribuição normal. Quanto a homocedasticidade, o pressuposto de homogeneidade de variâncias/covariâncias foi atendido tanto para as variáveis individualmente como pelo teste multivariado (Tabela 1).

Tabela 1 - Resultados dos testes de normalidade e homocedasticidade univariados para as variáveis e multivariados para o conjunto de variáveis do carvão vegetal dos clones de Corymbia e Eucalyptus.

\begin{tabular}{lll}
\hline \multicolumn{1}{c}{ Variável } & \multicolumn{1}{c}{ Teste } & Valor-p \\
\hline Rendimento da carbonização & Shapiro-Wilk & $0,0313^{\mathrm{ns}}$ \\
Teor de materiais voláteis & Shapiro-Wilk & $0,0068^{*}$ \\
Carbono fixo & Shapiro-Wilk & $0,1432^{\mathrm{ns}}$ \\
Friabilidade & Shapiro-Wilk & $0,3305^{\mathrm{ns}}$ \\
Densidade relativa aparente & Shapiro-Wilk & $0,0208^{\mathrm{ns}}$ \\
Poder calorífico superior & Shapiro-Wilk & $0,1403^{\mathrm{ns}}$ \\
Rendimento da carbonização & Bartlett & $0,2279^{\mathrm{ns}}$ \\
Teor de materiais voláteis & Bartlett & $0,0135^{\mathrm{ns}}$ \\
Carbono fixo & Bartlett & $0,3621^{\mathrm{ns}}$ \\
Friabilidade & Bartlett & $0,6243^{\mathrm{ns}}$ \\
Densidade relativa aparente & Bartlett & $0,5391^{\mathrm{ns}}$ \\
Poder calorífico superior & Bartlett & $0,3437^{\mathrm{ns}}$ \\
Conjunto das variáveis & Assimetria multivariada (Mardia) & $0,0009^{*}$ \\
Conjunto das variáveis & Curtose multivariada (Mardia) & $0,2671^{\mathrm{ns}}$ \\
Conjunto das variáveis & Homocedasticidade multivariada (Levene) & $0,6099^{\mathrm{ns}}$ \\
\hline
\end{tabular}

*Significativo a 1\%; ns: não significativo.

Ainda na análise preliminar, foi constatada a presença de sete outliers multivariados considerando o quantil ajustado de 16,64 da distribuição qui-quadrado. A presença de outliers pode resultar na rejeição da suposição de normalidade multivariada e gerar uma distribuição normal multivariada contaminada (Barbosa et al., 2018), o que afeta todas as inferências posteriores baseadas no pressuposto de normalidade (Schwager \& Margolin, 1982). Entretanto, a maior parte dos outliers são observações de um dos clones, ou seja, é indício de um material genético superior em termos de propriedades do carvão, sendo de interesse da pesquisa manter esses outliers no banco de dados.

Após a realização da análise de variância multivariada (MANOVA), verificou-se diferença significativa no campo multivariado com relação à qualidade do carvão vegetal produzido a partir da madeira dos diferentes clones de eucalipto (Tabela 2). O valor aproximado da estatística $\mathrm{F}^{\prime}$ para o teste de Wilks, assim como para os demais testes, foi altamente significativo (valor-p < 0,001), evidenciando a diferença de potencial entre os clones para a produção de carvão. 
Tabela 2 - Resumo da MANOVA para seis variáveis de rendimento e qualidade do carvão vegetal em função dos clones do gênero Corymbia e Eucalyptus.

\begin{tabular}{lccccc}
\hline \multicolumn{1}{c}{ Teste } & Estatística & F' $^{\prime}$ & GL(num) & GL(den) & Valor-p \\
\hline Wilks & 0,0177 & 3,7450 & 66 & 219,4900 & $<0,0001^{* * *}$ \\
Pillai & 2,5058 & 2,9277 & 66 & 270,0000 & $<0,0001 * * *$ \\
Hotelling-Lawley & 7,8345 & 7,8345 & 66 & 230,0000 & $<0,0001^{* * *}$ \\
Roy & 3,9556 & 16,1820 & 11 & 45,0000 & $<0,0001 * * *$ \\
\hline
\end{tabular}

F': valor aproximado da estatística F; GL(num): graus de liberdade do numerador; GL(den): graus de liberdade do denominador; $* * *$ Significativo a $0,1 \%$.

Procedendo a decomposição espectral da matriz resultante de $\mathbf{E}^{-1} \mathbf{H}$, foi constatado que o maior autovalor reteve aproximadamente $49 \%$ da informação contida nos dados (Tabela 3). Gomes (2009) afirmou que é possível utilizar apenas a primeira função discriminante (associada ao maior autovalor) para transformar as variáveis e explicar a variabilidade dos dados, desde que a retenção da informação pela primeira função seja suficiente. Fazendo um paralelo com análise fatorial e de componentes principais para redução eficiente da dimensionalidade de dados, Hair Junior et al. (2009) sugeriu retenção de ao menos $60 \%$ da variância total, enquanto Ferreira (2011) indicou 70\% ou mais. Como a primeira função discriminante reteve menos da metade da informação dos dados, foi utilizada também a segunda função discriminante para analisar os dados, agregando, assim, quase $80 \%$ da informação total.

Tabela 3 - Informação retida pelos seis autovalores referentes às seis respectivas variáveis avaliadas do carvão vegetal dos clones de Corymbia e Eucalyptus.

\begin{tabular}{|c|c|c|c|c|c|c|}
\hline \multirow{2}{*}{ Autovalor $(\lambda)$} & $\lambda_{1}$ & $\lambda_{2}$ & $\lambda_{3}$ & $\lambda_{4}$ & $\lambda_{5}$ & $\lambda_{6}$ \\
\hline & 3,8648 & 2,4469 & 0,9430 & 0,3183 & 0,2152 & 0,1111 \\
\hline Informação retida (\%) & 48,94 & 30,99 & 11,91 & 4,03 & 2,72 & 1,40 \\
\hline Informação acumulada (\%) & 48,94 & 79,93 & 91,84 & 95,87 & 98,59 & 100,00 \\
\hline
\end{tabular}

Selecionando-se e normalizando os autovetores da primeira e segunda coluna da matriz de autovetores referentes a matriz $\mathbf{E}^{-1} \mathbf{H}$, obteve-se os coeficientes normalizados da primeira e da segunda função discriminante de Fisher (Tabela 4). Conforme supracitado, por estarem associadas aos dois maiores autovalores, as duas primeiras funções discriminantes, juntas, explicam aproximadamente $80 \%$ da variabilidade dos dados, bem como maximizam a diferenciação dos tratamentos, neste caso os clones de Corymbia e Eucalyptus. Nota-se, ainda, que o maior coeficiente, em valor absoluto, é relacionado a variável carbono fixo no caso da primeira função, enquanto na segunda função o maior foi da densidade relativa aparente.

Tabela 4 - Coeficientes normalizados da primeira função discriminante (FD1) e da segunda função discriminante (FD2) de Fisher para as variáveis do carvão vegetal dos clones de Corymbia e Eucalyptus.

\begin{tabular}{cllccc}
\hline & \multicolumn{1}{c}{ Variável } & \multicolumn{2}{c}{ Coeficiente FD1 } & \multicolumn{2}{c}{ Coeficiente FD2 } \\
\hline$X_{1}$ & Rendimento da carbonização & $b_{1}$ & 0,4570 & $b_{1}$ & $0,2924.10^{-1}$ \\
$X_{2}$ & Teor de materiais voláteis & $b_{2}$ & 0,5438 & $b_{2}$ & 0,4180 \\
$X_{3}$ & Carbono fixo & $b_{3}$ & 0,6762 & $b_{3}$ & 0,4251 \\
$X_{4}$ & Friabilidade & $b_{4}$ & $-0,1842$ & $b_{4}$ & 0,2318 \\
$X_{5}$ & Densidade relativa aparente & $b_{5}$ & $-0,0654$ & $b_{5}$ & $-0,7681$ \\
$X_{6}$ & Poder calorífico superior & $b_{6}$ & $-0,9279.10^{-3}$ & $b_{6}$ & $0,3263.10^{-3}$ \\
\hline
\end{tabular}

*Variável canônica. 
A magnitude em módulo dos coeficientes da função discriminante pode ser utilizada como indicador de quais variáveis são mais importantes para a discriminação dos tratamentos (Simeão \& Padovani, 2008; Souza et al., 2009). Neste caso, a variável carbono fixo e densidade relativa aparente foram identificados como os principais atributos de qualidade do carvão vegetal para diferenciar os clones de eucalipto.

O carbono fixo do carvão vegetal é considerado um dos principais parâmetros para a avaliação de qualidade, sendo influenciada pelo material de origem e pelo processo de carbonização (Neves, 2012). Destaca-se que o teor de materiais voláteis, segundo Oliveira et al. (2010), é normalmente inversamente proporcional ao índice de carbono fixo do carvão, o que corrobora e sustenta a coerência dos resultados constatados, uma vez que os coeficientes dessas variáveis apresentaram magnitudes semelhantes em ambas as funções discriminantes.

A maximização da discriminação dos clones pela análise multivariada fica evidente ao analisar a Tabela 5, na qual nota-se a formação de 4 "grupos" distintos para as duas variáveis canônicas, de acordo com o teste de Scott-Knott. A qualidade global do carvão vegetal do clone AMF1119 (E. grandis $\times$ E. urophylla) foi superior aos demais. Destaca-se que parte dos outliers multivariados detectados foram justamente as observações referentes ao clone AMF1119, confirmando a superioridade desse clone.

Tabela 5 - Médias das variáveis canônicas geradas a partir de seis variáveis do carvão vegetal de clones de Corymbia (M02 a M22) e Eucalyptus (AMF1119).

\begin{tabular}{cccc}
\hline Clone & Variável canônica 1 & Clone & Variável canônica 2 \\
\hline AMF1119 & $73,0840 a *$ & AMF1119 & $46,6979 a$ \\
M02 & $72,3399 b$ & M43 & $46,4865 a$ \\
M04 & $72,0753 b$ & $M 22$ & $46,3313 b$ \\
M01 & $71,9164 b$ & M06 & $46,1968 b$ \\
M06 & $71,8650 b$ & M08 & $46,0822 c$ \\
M03 & $71,8545 b$ & M09 & $46,0304 c$ \\
M08 & $71,8506 b$ & $M-S / N$ & $46,0255 c$ \\
M09 & $71,5499 c$ & M07 & $46,0225 c$ \\
M07 & $71,5338 c$ & M04 & $45,8170 d$ \\
M-S/N & $71,0537 d$ & M02 & $45,7817 d$ \\
M43 & $71,0478 d$ & M01 & $45,7817 d$ \\
M22 & $70,7581 d$ & $M 03$ & $45,6111 d$
\end{tabular}

*Clones com médias seguidas de mesma letra pertencem ao mesmo grupo, pelo teste Scott-Knott a 1\% de significância.

É importante observar que o clone AMF1119 embora tenha sido superior pelas médias canônicas, apresenta teor de materiais voláteis e friabilidade sutilmente acima da média geral dos clones, bem como densidade aparente abaixo da média (Tabela 6). Os clones M02 e M43, ambos híbridos de C. torelliana x C. citriodora, se destacam em relação aos demais do gênero Corymbia na classificação pela variável canônica, fato que se deve principalmente aos seus resultados de friabilidade e poder calorífico superior, respectivamente. As variações entre clones de um mesmo tipo de híbrido evidenciam a importância do refinamento do processo de melhoramento genético, visto que mesmo entre genótipos teoricamente similares, podem ser obtidas consideráveis diferenças entre clones para características de interesse. 
Tabela 6 - Médias do rendimento da carbonização (Rend), teor de materiais voláteis (Volát), carbono fixo (Carb), friabilidade (Friab), densidade aparente (Dens) e poder calorífico superior (PCS) de clones de Corymbia (M01 a M-S/N) e Eucalyptus (AMF1119).

\begin{tabular}{ccccccc}
\hline Clone & Rend(\%) & Volát(\%) & Carb(\%) & Friab(\%) & Dens(g cm $\left.\mathbf{~}^{-3}\right)$ & PCS(kcal $\mathbf{~ g}^{-1}$ ) \\
\hline AMF1119 & 38,21 & 28,79 & 70,61 & 6,40 & 0,35 & 7084,40 \\
M01 & 36,23 & 28,67 & 69,70 & 5,02 & 0,41 & 6910,60 \\
M02 & 37,50 & 28,68 & 69,49 & 4,93 & 0,33 & 6952,00 \\
M03 & 36,82 & 28,24 & 69,66 & 4,77 & 0,37 & 7032,75 \\
M04 & 36,80 & 27,81 & 70,33 & 5,68 & 0,43 & 6840,00 \\
M06 & 36,84 & 27,79 & 70,50 & 6,59 & 0,38 & 7021,40 \\
M07 & 36,35 & 28,07 & 70,15 & 5,98 & 0,41 & 7160,40 \\
M08 & 36,35 & 27,79 & 70,53 & 6,24 & 0,36 & 6884,40 \\
M09 & 35,85 & 26,93 & 71,28 & 5,72 & 0,30 & 7118,80 \\
M22 & 35,40 & 27,85 & 70,28 & 7,17 & 0,33 & 7264,60 \\
M43 & 35,72 & 27,18 & 71,11 & 7,60 & 0,35 & 7234,25 \\
M-S/N & 36,05 & 27,20 & 70,45 & 6,89 & 0,33 & 7065,17 \\
Média & 36,51 & 27,92 & 70,34 & 6,08 & 0,36 & 7047,40 \\
Média C* & 36,36 & 27,84 & 70,32 & 6,05 & 0,36 & 7044,03 \\
\hline
\end{tabular}

*Média geral apenas dos clones de Corymbia, excluindo o clone AMF1119.

Os clones do gênero Corymbia, apesar de serem inferiores ao clone de Eucalyptus nos resultados desta pesquisa, apresentaram em geral desempenho bastante interessante, o que reforça o potencial do híbrido $C$. torelliana $\times$ C. citriodora para a produção de carvão. A média de rendimento gravimétrico geral (36,36\%) dos clones Corymbia ultrapassou o rendimento gravimétrico de seis dos oito clones de Eucalyptus analisados no trabalho de Moutinho et al. (2017), porém ficou consideravelmente abaixo dos valores dos dois clones de Corymbia da mesma pesquisa. Comparando com a catingueira (Poincianella pyramidalis) e pau-d'arco (Handroanthus impetiginosus) estudadas por Medeiros Neto et al. (2014), os resultados dos clones de Corymbia foram melhores quanto ao teor de materiais voláteis, carbono fixo e poder calorífico superior, porém, piores em relação ao rendimento gravimétrico e densidade aparente. Em relação a friabilidade, a média dos híbridos C. torelliana $\times$ C. citriodora foi menor que do Eucalyptus saligna (Coutinho \& Ferraz, 1988) e de espécies amazônicas (Silva, 1988).

As variáveis canônicas representaram de forma mais eficiente a variabilidade em função dos clones, de modo que o valor da estatística $\mathrm{F}$ destas variáveis é consideravelmente maior que os valores para as demais variáveis, especialmente da primeira variável canônica. A acurácia seletiva das variáveis canônicas também superou as demais, ratificando que a função discriminante é melhor para a discriminação dos clones avaliados (Tabela 7).

Tabela 7 - Valores da estatística F, valor-p e acurácia seletiva (AS) das variáveis observadas em análise univariada e variáveis canônicas para os clones de Corymbia e Eucalyptus.

\begin{tabular}{lccc}
\hline Variável & F & valor-p & AS (\%) \\
\hline Rendimento da carbonização & 5,165 & $3,45.10^{-5}$ & 89,80 \\
Teor de materiais voláteis & 1,375 & 0,22 & 52,22 \\
Carbono fixo & 1,110 & 0,38 & 31,48 \\
Friabilidade & 5,956 & $7,01.10^{-6}$ & 91,22 \\
Densidade relativa aparente & 4,124 & $3,28.10^{-4}$ & 87,04 \\
Poder calorífico superior & 2,677 & $9,83.10^{-3}$ & 79,15 \\
Variável canônica 1 & 16,040 & $3,95.10^{-12}$ & 97,34 \\
Variável canônica 2 & 9,459 & $1,66.10^{-8}$ & 94,57 \\
\hline
\end{tabular}




\section{CONCLUSÃO}

A função discriminante de Fisher foi eficiente na sumarização dos dados quando utilizadas as duas primeiras variáveis canônicas, retendo aproximadamente $80 \%$ da informação contida no conjunto de seis variáveis. Em comparação às análises univariadas, o uso da função discriminante para gerar novas variáveis que são funções das variáveis originais apresenta vantagens quanto a simplificação e clareza na diferenciação dos clones em relação à qualidade global do carvão vegetal.

As variáveis de maior influência na discriminação do carvão produzido pelos diferentes clones foram carbono fixo e densidade relativa aparente do carvão. Comparando os clones por meio das variáveis canônicas constata-se que o clone de Eucalyptus grandis x Eucalyptus urophylla, que já é frequentemente utilizado em plantios com finalidade energética e outras, mantem-se como a melhor opção para a produção de carvão vegetal dentro do conjunto de genótipos analisados. Não obstante, os clones do gênero Corymbia apresentaram, em geral, resultados promissores para a sua utilização em plantios destinados à produção de carvão.

\section{AGRADECIMENTOS}

À Coordenação de Aperfeiçoamento de Pessoal de Nível Superior - CAPES pelo apoio em forma de concessão de bolsa de pós-graduação - Código de Financiamento 001 e à empresa ArcelorMittal BioFlorestas pelo compartilhamento de dados experimentais.

\section{REFERÊNCIAS}

Anderson, M. J. (2006). Distance-based tests for homogeneity of multivariate dispersions. Biometrics, 62(1), 245-253. PMid:16542252. http://dx.doi.org/10.1111/j.1541-0420.2005.00440.x.

Associação Brasileira de Normas Técnicas - ABNT. (1984). NBR 8633: carvão vegetal: determinação do poder calorífico (13 p.). Rio de Janeiro: ABNT.

Associação Brasileira de Normas Técnicas - ABNT. (1986). NBR 8112: carvão vegetal: análise imediata (8p.). Rio de Janeiro: ABNT.

Associação Brasileira de Produtores de Florestas Plantadas - ABRAF. (2013). Anuário estatístico ABRAF 2013 ano base 2012 (148 p.). Brasília: ABRAF.

Barbosa, J. J., Pereira, T. M., \& Oliveira, F. L. P. (2018). Uma proposta para identificação de outliers multivariados. Ciência e Natura, 40, 40. http://dx.doi.org/10.5902/2179460X29535.

Campos, K. A. (2012). Função discriminante de Fisher como alternativa à análise de variância multivariada (Tese de doutorado). Universidade Federal de Lavras, Lavras,

Castro, A. F. N. M., Castro, R. V. O., Carneiro, A. C.O., Lima, J. E., Santos, R. C., Pereira, B. L. C., \& Alves, I. C. N. (2013). Análise multivariada para seleção de clones de eucalipto destinados à produção de carvão vegetal. Pesquisa Agropecuária Brasileira, 48(6), 627-635. http://dx.doi.org/10.1590/S0100204X2013000600008.

Coutinho, A. R., \& Ferraz, E. S. B. (1988). Determinação da friabilidade do carvão vegetal em função do diâmetro das árvores e temperatura de carbonização. IPEF, (38), 33-37.

Ferreira, D. F. (2011). Estatística multivariada (2. ed. rev. e ampl., 675 p.). Lavras: UFLA.

Fisher, R. A. (1936). The use of multiple measurements in taxonomic problems. Annals of Eugenics, 7(2), 179-188. http://dx.doi.org/10.1111/j.1469-1809.1936.tb02137.x.

Food and Agriculture Organization - FAO. (2017). The charcoal transition: greening the charcoal value chain to mitigate climate change and improve local livelihoods. Rome: FAO.

Gomes, F. P. (2009). Curso de estatística experimental (15. ed., 451 p.). Piracicaba: ESALQ.

Goulart, M., Haselein, C. R., Hoppe, J. M., Farias, J. A., \& Pauleski, D. T. (2003). Massa específica básica e massa seca de madeira de Eucalyptus grandis sob o efeito do espaçamento de plantio e da posição axial no tronco. Ciência Florestal, 13(2), 167-175. http://dx.doi.org/10.5902/198050981753.

Hair Junior, J. F., Black, W. C., Babin, B. J., Anderson, R. E., \& Tatham, R. L. (2009). Multivariate data analysis (7th ed., 785 p.). Upper Saddle River: Prentice Hall.

Harris, R. J. (1975). A primer of multivariate statistics. New York: Academic Press. 
Indústria Brasileira de Árvores - IBÁ. (2018). Dados do relatório IBÁ 2018: ano base 2017. Brasília. Recuperado em 7 de março de 2019, de https://www.iba.org/datafiles/publicacoes/relatorios/digitalsumarioexecutivo-2018.pdf

Mardia, K. V. (1970). Measures of multivariate skewness and kurtosis with applications. Biometrika, 57(3), 519-530. http://dx.doi.org/10.1093/biomet/57.3.519.

Mardia, K. V. (1974). Applications of some measures of multivariate skewness and kurtosis for testing normality and robustness studies. Sankhya, 36(2), 115-128.

Mardia, K. V. (1975). Assessment of multinormality and the robustness of Hotelling's $T^{2}$ test. Applied Statistics, 24(2), 163-171. http://dx.doi.org/10.2307/2346563.

Medeiros Neto, P. N., Oliveira, E., \& Paes, J. B. (2014). Relações entre as características da madeira e do carvão vegetal de duas espécies da Caatinga. Floresta e Ambiente, 21(4), 484-493. http://dx.doi.org/10.1590/2179-8087.051313.

Mingoti, S. A. (2005). Análise de dados através de métodos de estatística multivariada: uma abordagem aplicada. Belo Horizonte: Editora UFMG.

Motta, L. P., Machado, C. C., Soares, V. P., Ribeiro, J. C., \& Ribeiro, C. A. A. S. (1996). Utilização de sistema de informações e da distância virtual na otimização do transporte florestal rodoviário. Revista Árvore, 20(3), 381-394.

Moutinho, V. H. P., Tomazello Filho, M., Brito, J. O., Ballarin, A. W., Andrade, F. W. C., \& Cardoso, C. (2017). Characterization and statistical correlation between charcoal's physical and mechanical properties of Eucalyptus and Corymbia clones. Ciência Florestal, 27(3), 1095-1103. http://dx.doi.org/10.5902/1980509828684.

Neves, T. A. (2012). Qualidade da madeira e do carvão vegetal de clones de Eucalyptus cultivados no Sul de Minas Gerais (Dissertação de mestrado). Universidade Federal de Lavras, Lavras.

Oliveira, A. C., Carneiro, A. C.O., Vital, B. R., Almeida, W., Pereira, B. L. C., \& Cardoso, M. T. (2010). Parâmetros de qualidade da madeira e do carvão vegetal de Eucalyptus pellita F. Muell. Scientia Forestalis, 38(87), 431-439.

Oliveira, J. B., Vivacqua Filho, A., Mendes, M. G., \& Gomes, P. A. (1982). Produção de carvão vegetal: aspectos técnicos. In Fundação Centro Tecnológico de Minas Gerais - CETEC (Ed.), Produção $e$ utilização de carvão vegetal (pp. 59-72). Belo Horizonte: CETEC.

Protásio, T. P., Couto, A. M., Reis, A. A., \& Trugilho, P. F. (2013). Seleção de clones de Eucalyptus para a produção de carvão vegetal e bioenergia por meio de técnicas univariadas e multivariadas. Scientia Forestalis, 41(97), 15-28.

Protásio, T. P., Trugilho, P. F., Neves, T. A., \& Vieira, C. M. M. (2012). Análise de correlação canônica entre características da madeira e do carvão vegetal de Eucalyptus. Scientia Forestalis, 40(95), 317-326.

R Core Team. (2018). R: a language and environment for statistical computing. Vienna: R Foundation for Statistical Computing.

Resende, M. D. V. (2002). Genética biométrica e estatística no melhoramento de plantas perenes (975 p.). Brasília: Embrapa Informação Tecnológica.

Schwager, S. J., \& Margolin, B. H. (1982). Detection of multivariate normal outliers. Annals of Statistics, 10(3), 943-954. http://dx.doi.org/10.1214/aos/1176345884.

Silva, D. A. (1988). Qualidade do carvão vegetal produzido com madeiras da região de Manaus em fornos de alvenaria. Acta Amazonica, 18(1-2), 163-178. http://dx.doi.org/10.1590/1809-43921988182178.

Simeão, S. F. A. P., \& Padovani, C. R. (2008). Utilização da função discriminante quadrática em ciências experimentais. Revista Energia na Agricultura, 23(1), 116-134.

Souza, U. R., Silva, F. L., Griffith, J. J., Lima, J. E., Quintela, M. C. A., \& Costa, E. C. V. (2009). Determinantes dos novos contratos de fomento florestal na Mesorregião do Vale do Rio Doce, Minas Gerais. Revista Árvore, 33(2), 377-386. http://dx.doi.org/10.1590/S0100-67622009000200019.

Trugilho, P. F., Lima, J. T., Mori, F. A., \& Lino, A. L. (2001). Avaliação de clones de Eucalyptus para produção de carvão vegetal. Cerne, 7(2), 104-114.

Vital, B. R. (1984). Métodos de determinação da densidade da madeira (21р.). Viçosa: Sociedade de Investigações Florestais.

Contribuição dos Autores: KDP: conceituação, análise formal, escrita - primeira redação; APSC: conceituação, supervisão, ACOC: investigação, validação; GRS: metodologia e LFF: escrita - revisão e edição. 